\title{
Assessment of lung cancer mortality reduction after chest X-ray screening in smokers: A population-based cohort study in Varese, Italy
}

\author{
Lorenzo Dominioni $^{\mathrm{a}, *}$, Albino Poli ${ }^{\mathrm{b}}$, William Mantovani ${ }^{\mathrm{b}}$, Salvatore Pisani ${ }^{\mathrm{c}}$, Nicola Rotolo $^{\mathrm{a}}$, \\ Massimo Paolucci ${ }^{\mathrm{d}}$, Fausto Sessa ${ }^{\mathrm{e}}$, Valentina Conti ${ }^{\mathrm{a}}$, Vincenzo D’Ambrosio ${ }^{\mathrm{f}}$, Antonio Paddeu ${ }^{\mathrm{g}}$, \\ Andrea Imperatori ${ }^{\mathrm{a}}$
}

a Center for Thoracic Surgery, University of Insubria, Ospedale di Circolo, Varese, Italy

${ }^{\mathrm{b}}$ Department of Public Health and Community Medicine, University of Verona, Verona, Italy

${ }^{\mathrm{c}}$ Epidemiology Observatory, Azienda Sanitaria Locale, Varese, Italy

d Department of Radiology, Ospedale S. Antonio Abate, Gallarate, Italy

e Department of Surgical and Morphological Sciences, University of Insubria, Varese, Italy

${ }^{\mathrm{f}}$ Thoracic Medicine Unit, Ospedale S. Antonio Abate, Gallarate, Italy

${ }^{\mathrm{g}}$ Respiratory Care Unit, Ospedale S. Anna, San Fermo della Battaglia, Como, Italy

\section{A R T I C L E I N F O}

Article history:

Received 4 October 2012

Accepted 10 December 2012

\section{Keywords:}

Lung cancer

Mortality

Smoking

Cohort study

Chest X-ray screening

Population-based

\begin{abstract}
A B S T R A C T
Background: The effectiveness of screening for lung cancer (LC) in smokers on a population level, as distinct from the special circumstances that may apply in a randomized trial of selected volunteers, has not been thoroughly investigated. Here we evaluate by the standardized mortality ratio (SMR) indicator the impact of a chest X-ray (CXR) screening programme carried out at community level on LC mortality in smokers.

Methods: All smokers of $>10$ pack-years, of both genders, ages $45-75$ years, resident in 50 communities of the Province of Varese, Italy, screening-eligible, in 1997 were invited by their National Health Service (NHS) general practitioner physicians to a nonrandomized programme of five annual CXR screenings. The entire invitation-to-screen cohort ( $n=5815$ subjects) received NHS usual care, with the addition of CXR exams in volunteer participants (21\% of invitees), and was observed through December 2006. To overcome participants' selection bias of LC mortality assessment, for the entire invitation-to-screen cohort we estimated the LC-specific SMR, based on the local reference population receiving the NHS usual care.

Results: Over the 8-year period 1999-2006, a total of 172 cumulative LC deaths were observed in the invitation-to-screen cohort; 210 were expected based on the reference population. Each year in the invited cohort the observed LC deaths were fewer than expected. The cumulative LC SMR was 0.82 (95\%CI, $0.67-0.99 ; p=0.048$ ), suggesting that LC mortality was reduced by $18 \%$ with CXR screening.

Conclusion: Implementation of a CXR screening programme at community level was associated with a significant reduction of LC mortality in smokers.
\end{abstract}

(c) 2012 Elsevier Ireland Ltd. All rights reserved.

\section{Introduction}

Lung cancer (LC) is a relevant public health problem worldwide. Beyond prevention and cessation of cigarette smoking, other strategies are needed, because most who will succumb to this disease are current or former smokers [1]. No public policy organization recommends chest X-ray (CXR) screening, as randomized studies showed no LC mortality benefit [2-6]. However, the published

\footnotetext{
* Corresponding author at: Center for Thoracic Surgery, University of Insubria, Ospedale di Circolo, Via Guicciardini, 21100 Varese, Italy. Tel.: +39 0332 278868; fax: +390332 260260

E-mail address: lorenzo.dominioni@uninsubria.it (L. Dominioni).
}

randomized CXR screening trials present relevant methodological weaknesses $[1,4,7,8]$; moreover, these studies were performed in highly selected volunteers $[2,3,5]$, and the external value of their results remains uncertain. Notably, the U.S. Preventive Service Task Force concluded that the evidence is insufficient to recommend for or against screening with CXR [9]. The effectiveness of screening for LC in the community, as distinct from the special circumstances that may apply in a randomized trial of volunteers, has not been thoroughly investigated and remains controversial [10]. With the aim to assess whether radiographic screening of smokers in the community setting in Italy decreases LC mortality, in 1997 we started a nonrandomized observational study of CXR screening in the PREDICA cohort [11]. The latter is a clearly defined populationbased cohort of smokers of the Varese Province, hereafter referred 
to as "cohort", that was invited to an annual CXR screening programme and prospectively followed up. We previously reported the cohort's demographic characteristics, screening protocol, participants adherence, and LC detection results [11,12]. After completion of the CXR screening programme, here we evaluate its effectiveness in the cohort by estimating the standardized mortality ratio (SMR), an indicator recently used by other investigators to document the significant LC mortality reduction achieved by computed tomography (CT) screening of smokers [13].

\section{Materials and methods}

\subsection{Study design}

This is a prospective nonrandomized population-based cohort study of CXR screening for LC, with a comparison group consisting of the reference population of the screening catchment geographical district. The cohort, made of the totality of residents in 50 communities of the Varese Province who were screening-eligible cigarette smokers, was invited to annual CXR screening for 5 years and was observed for 9.5 years. In this geographical area no other LC screening projects were ongoing. At the end of study, we evaluated the impact of screening on LC mortality in the cohort. To this effect, as our endpoint we estimated the cumulative LC SMR, expressed as the ratio of total LC deaths observed in the entire screening-invited cohort to total LC deaths expected based on the local reference population receiving usual care. This intention-to-treat approach for analysis of the screening programme effectiveness overcomes the participants' selection bias $[14,15]$.

\subsection{The cohort}

We aimed to recruit a population-based cohort of approximately 5000 smokers, a sample size similar to that of the intervention arm in the Kaiser Permanente trial [16] and in the Mayo Lung Project [2]. Recruitment of the cohort was effected by a pool of 50 general practitioner (GP) physicians of the Italian National Health Service (NHS), as previously described [11]. Notably, the medical practices of these GPs were located in 50 communities scattered over rural and urban areas, a large sample of the total 230 communities of the 44-town screening catchment area in the Varese Province [11]. Briefly, based on practice records, in early 1997 the GPs invited to a CXR screening programme the totality of smokers resident and registered in their community practice who possessed all the following inclusion criteria: both genders, birth cohort 1923-1953, current or ex-smoker of $>10$ pack-years. Exclusion criteria were: subject unfit for surgery or with diagnosed/suspected LC. After exclusion of 110 noneligible candidates, 5815 subjects featuring a median smoking history of 32.8 pack-years [interquartile range (IQR), 22.8-46.0], constituted the population-based cohort of this study. The screening programme was offered free of charge and consisted of a baseline two-view CXR exam and an annual repeat screen for 4 years. The 1244 individuals (21\% of the cohort) who accepted the invitation undertook the baseline CXR examination during a 4.5-year recruitment period (from July 1997 through December 2001, median in February 1998) and were defined as participants in screening. All participants signed an informed consent form; for nonparticipants (79\% of the cohort), informed consent was waived. In addition, during 1997-2001 a total of 1221 uninvited screeningeligible individuals (resident in the 44-town catchment area, but outside the 50 communities of cohort recruitment) requested to participate after learning about the screening programme. Also these additional candidates signed informed consent, and undertook the screening protocol, but they constituted a distinct group of "uninvited participants" that will be evaluated in a separate study.
All names were encrypted, as approved by the Varese Hospital and Health District Ethics Committee. The data collected in this study were stored in a secure database at the Center for Thoracic Surgery, University of Insubria.

\subsection{Standard of care, screening and follow-up of the cohort}

The NHS usual care was provided to the entire cohort. In addition, participants underwent screening by chest radiographs, according to the previously described protocol [12]. The study lasted from July 1, 1997 until December 31, 2006 and during this interval the entire cohort was observed. After baseline screening, the 1244 participants underwent a total of 4337 annual repeat screens. The standard of care in Varese was used to investigate suspicious screen-detected or nonscreen-detected CXR abnormalities, without special algorithm. Management of LCs was centralized in the Varese University Hospital, and cases were treated by usual international criteria [17] regardless of mode of LC detection. At study cut off, the vital status of $98.5 \%$ of cohort individuals were ascertained by linkage with the Lombardy Health Registry of all residents in the Lombardy Region. At study cut off 85 subjects (1.5\% of cohort) were untraceable. In deceased cohort subjects, death certificates were used to identify LC as the cause of death, by linkage with the Varese Province Mortality Registry, after review by the mortality review committee of this study (LD, AI, NR, FS, APo, WM), as previously described [11]. Deaths attributed to LC treatment complications were filed as deaths from LC. The LC deaths that occurred in the cohort were recorded by gender and age strata.

\subsection{Lung cancer standardized mortality ratio}

We excluded from LC mortality analysis the initial period of study, from July 1997 through December 1998, as we presumed that screening was unlikely to decrease LC mortality immediately. Moreover, exclusion of this initial 18-month period minimizes the healthy-cohort recruitment bias, i.e. an artificially low LC mortality in the cohort due to enrolment of asymptomatic subjects [18]. Furthermore, a preliminary joinpoint analysis [19] identified in 1999 as the year during which a statistically significant mortality trend inflexion occurred in the cohort. Accordingly, we confined the analysis of LC SMR in the cohort to the 8-year interval between January 1, 1999 and December 31, 2006. This timeframe was chosen based on the suggestion to add about 4 years after the end of active screening (median in 2002 in our study) to evaluate the effectiveness of screening using LC mortality [5]. Our analysis was not extended over a longer period, because any benefit from short-term screening is likely transient [8]. To calculate the cumulative LC SMR of the cohort during 1999-2006, the ratio's numerator (number of observed LC deaths) was available from the cohort follow-up, while the denominator (number of expected LC deaths) was calculated as follows:

(a) First, we identified the source population of the cohort alive as of January 1,1999, constituted by all individuals of birth cohort 1923-1953 who were alive by that date and resident in all 230 communities of the 44-town catchment area [11] (Fig. 1). This source population was searched by linkage with the Varese Province general population registry, and consisted of 122,074 persons, shown by strata in Table 1.

(b) The reference population, constituted by all persons of the above source population possessing the smoking criteria for screening, was constructed multiplying the number of individuals in the source population strata by the corresponding prevalence rates of smokers (current or former smokers of $>10$ packyears), available from the year 2000 Varese Province population 


\section{Source population $(n=122,074)$}

\section{Inclusion criteria}

All persons of birth cohort 1923-1953 resident as of January 1, 1999 in the 230 communities of the 44town catchment area of screening in the Varese Province [11]

\section{Reference population $(n=26,656)$}

\section{Inclusion criteria}

All source population persons who were current or ex-smokers of $>10$ pack-years

Fig. 1. Composition of the source population and of the reference population as of January 1,1999 . The reference population was constructed multiplying the number of individuals in the source population strata by the prevalence rates of smokers (current or ex-smokers of $>10$ pack-years) in the strata.

Table 1

Source population of the cohort as of January 1,1999 , by strata.

\begin{tabular}{|c|c|c|c|c|c|c|}
\hline \multirow[t]{2}{*}{ Age, years ${ }^{\mathrm{a}}$} & \multicolumn{2}{|c|}{ Men, $n(\%)$} & \multicolumn{2}{|c|}{ Women, $n(\%)$} & \multicolumn{2}{|c|}{ Total, $n(\%)$} \\
\hline & 57,232 & (46.9) & 64,842 & (53.1) & 122,074 & $(100)$ \\
\hline $45-49$ & 10,971 & (19.2) & 11,318 & (17.5) & 22,289 & (18.3) \\
\hline $50-54$ & 10,994 & (19.2) & 11,594 & (17.9) & 22,588 & (18.5) \\
\hline $55-59$ & 10,437 & (18.2) & 10,967 & (16.9) & 21,404 & (17.5) \\
\hline $60-64$ & 9387 & (16.4) & 10,727 & (16.5) & 20,114 & (16.5) \\
\hline $65-69$ & 7939 & (13.9) & 9570 & (14.8) & 17,509 & (14.3) \\
\hline $70-74$ & 6387 & (11.2) & 8867 & (13.7) & 15,254 & (12.5) \\
\hline 75-79 & 1117 & $(2.0)$ & 1799 & $(2.8)$ & 2916 & (2.4) \\
\hline
\end{tabular}

${ }^{a}$ Mean age was 58.7 (8.6 SD) in men, 59.6 (8.8) in women, 59.0 (8.7) in total. SD, standard deviation.

survey [20] (Table 2). In total 26,656 subjects formed the reference population.

(c) The vital status as of December 31, 2006 of $98.2 \%$ of the 122,074 source population individuals were ascertained by deterministic record linkage with the Varese Province population registry and with the Lombardy Health Registry of all residents in the Lombardy Region [21]. At study cut off, $1.8 \%$ of subjects of the source population were untraceable. All LC deaths which occurred

Table 2

Reference population by strata, estimated multiplying the source population strata (Table 1 ) by the proportion of smokers.

\begin{tabular}{llllcc}
\hline \multirow{2}{*}{ Age, years } & \multicolumn{2}{l}{ Proportion of smokers } & & \multicolumn{2}{c}{ Reference population } \\
\cline { 2 - 3 } \cline { 5 - 6 } & Men & Women & & Men, $n$ & Women, $n$ \\
\hline $45-49$ & 0.322 & 0.151 & & 3533 & 1709 \\
$50-54$ & 0.328 & 0.126 & & 3606 & 1461 \\
$55-59$ & 0.360 & 0.107 & & 3757 & 1173 \\
$60-64$ & 0.357 & 0.092 & & 3351 & 987 \\
$65-69$ & 0.366 & 0.081 & & 2906 & 775 \\
$70-74$ & 0.355 & 0.070 & & 2267 & 621 \\
$75-79$ & 0.344 & 0.070 & & 384 & 126 \\
Total & & & 19,804 & 6852 \\
\hline
\end{tabular}

Note. Median pack-years in the surveyed smokers [20] were 33.2 (IQR, 22.5-47.0), not significantly different from the median pack-years in the study cohort (32.8 [IQR, 22.8-46.0], $p=0.473$ ). IQR, interquartile range.

a Proportion of smokers of $>10$ pack-years in the 1923-1953 birth cohort of Varese Province population, as recorded in the year 2000 survey [20]. during 1999-2006 in the source population were searched using the Varese Cancer Registry criteria [22] based on death certificates (not verified by the mortality review committee of this study), by linkage with the Varese Province Mortality Registry [23]. The latter was accessed in 2008, completed with certificates of deaths occurring up to December 31, 2006. The LC deaths occurred in the source population were recorded by strata.

(d) Then, to estimate the number of LC deaths in the reference population strata, we multiplied the number of LC deaths in each gender and age stratum of the source population (c) by the proportion of smoking-attributable LC deaths (0.86). The latter is the most conservative value (range, 0.86-0.90) of the smoking-attributable proportion of LC deaths recorded during the interval 1999-2006 by the Varese Epidemiology Observatory in the population of birth cohort 1923-1953.

(e) Finally, to calculate the LC deaths expected in the cohort we multiplied the estimated number of LC deaths in each reference population stratum (d) by the ratio of cohort subjects alive as of January 1, 1999 to the number of reference population subjects in the stratum. The total number of LC deaths expected in the cohort was the sum of expected LC deaths in each stratum.

For the above calculations we assumed that no substantial changes occurred during 1999-2006 in the proportion of smokingattributable LC deaths and in the ratio of cohort subjects to reference population subjects.

\subsection{Statistical analysis}

Data are presented as frequency, percentage, mean or median, as appropriate; 95\% confidence interval (CI) and IQR are shown. Differences were tested by Student's $t$ test, chi square test, Fisher's exact test, or Mann-Whitney $U$ test as required by type and distribution of variables.

Joinpoint regression analysis was used to identify the point where a statistically significant change in linear slope of the mortality trend over the study period occurred [19]. The joinpoint analysis was performed with software from the surveillance research programme of the U.S. NCI [24].

The LC SMR was computed using STATA program version 11.2 (Stata Corporation, College Station, TX). Two-tailed $p$ values of $<0.05$ were considered significant. Analysis was performed at the Department of Public Health and Community Medicine, University of Verona. Approval of this study and permission to access and analyze the anonymized linked data reported in this paper was granted by the Varese Hospital and Health District Ethics Committee. This study is registered, ISRCTN90639073.

\section{Results}

Over the 8-year period 1999-2006, cumulatively 172 LC deaths were observed in the cohort, while 1405 LC deaths altogether were recorded in the source population (Table 3 ).

The LC deaths estimated in the reference population strata, and those expected in the cohort are shown in Table 3. In total 210 LC deaths were expected in the cohort. Each year during 1999-2006 the number of LC deaths observed in the cohort was lower than that expected based on the reference population (Fig. 2). During $1999-2006$, the cumulative LC SMR was $172 / 210=0.82(95 \% \mathrm{CI}$, $0.67-0.99 ; p=0.048)$. This result indicates that the risk of LC death for the smokers invited to the screening programme at community level was reduced by $18 \%$ compared to that of the reference population smokers receiving usual care. The numbers of observed and expected LC deaths by age in the cohort are given in Table 4. 
Table 3

Lung cancer deaths during 1999-2006, by strata.

\begin{tabular}{|c|c|c|c|c|c|c|c|c|c|}
\hline \multirow[t]{2}{*}{ Age, years } & \multicolumn{3}{|c|}{ Men, $n$} & \multicolumn{3}{|c|}{ Women, $n$} & \multicolumn{3}{|c|}{ Total, $n$} \\
\hline & $(\mathrm{A})$ & (B) & (C) & (A) & (B) & (C) & (A) & (B) & (C) \\
\hline $45-49$ & 43 & 37 & 8 & 19 & 16 & 3 & 62 & 53 & 11 \\
\hline $50-54$ & 85 & 73 & 15 & 39 & 34 & 4 & 124 & 107 & 19 \\
\hline 55-59 & 127 & 109 & 24 & 57 & 49 & 6 & 184 & 158 & 30 \\
\hline $60-64$ & 185 & 159 & 34 & 75 & 65 & 7 & 260 & 224 & 41 \\
\hline $65-69$ & 275 & 237 & 42 & 92 & 79 & 9 & 367 & 316 & 51 \\
\hline $70-74$ & 252 & 217 & 42 & 87 & 75 & 8 & 339 & 292 & 50 \\
\hline 75-79 & 48 & 41 & 7 & 21 & 18 & 1 & 69 & 59 & 8 \\
\hline Total & 1015 & 873 & 172 & 390 & 335 & 38 & 1405 & 1208 & 210 \\
\hline
\end{tabular}

Note. Lung cancer deaths: (A) observed in the source population; (B) estimated in the reference population; (C) expected in the cohort. (B) was estimated multiplying (A) by 0.86 (proportion of smoking-attributable LC deaths). (C) was estimated multiplying (B) by the ratio of cohort subjects to reference population subjects in the stratum. LC, lung cancer.
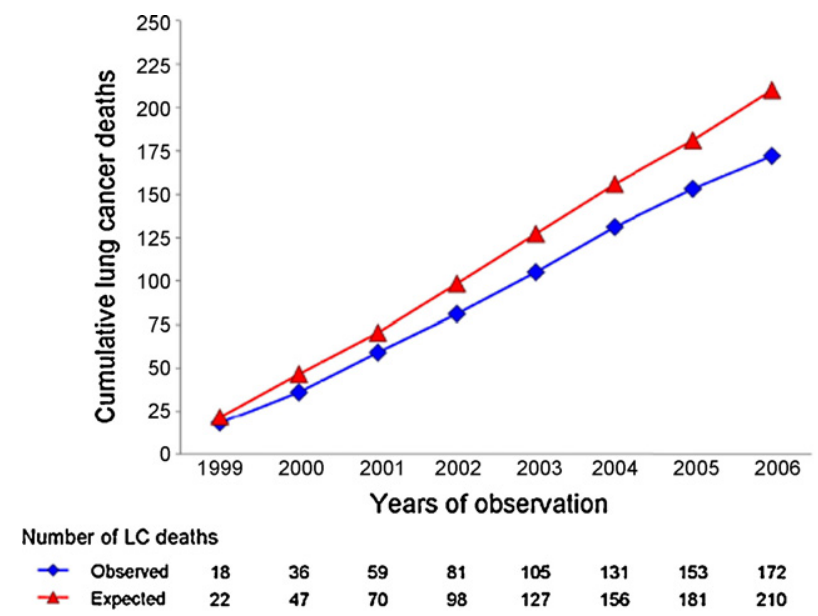

Fig. 2. Cumulative lung cancer deaths observed in the screening-invited cohort ( $\downarrow)$, and expected based on reference population receiving usual care $(\mathbf{\Delta})$.

\section{Discussion}

In this study we assessed the LC SMR in a well defined population-based cohort of smokers invited to a CXR screening programme at community level. After screening implementation we found that LC mortality over an 8-year period was significantly reduced in the entire invitation-to-screen cohort of smokers. In contrast, no substantial LC mortality benefit from screening with chest radiographs was shown in the PLCO cancer randomized trial after 13 years of follow-up [5]. These opposite results do not

Table 4

Observed and expected lung cancer (LC) deaths by age in the cohort.

\begin{tabular}{|c|c|c|c|}
\hline \multicolumn{2}{|l|}{ Cohort } & \multicolumn{2}{|c|}{$\begin{array}{l}\text { LC deaths in the cohort during } \\
1999-2006\end{array}$} \\
\hline Age, years & Rank population, $n$ & Observed, $n$ & Expected, $n$ \\
\hline $45-49$ & 1210 & 12 & 11 \\
\hline $50-54$ & 1187 & 16 & 19 \\
\hline $55-59$ & 1079 & 22 & 30 \\
\hline $60-64$ & 932 & 37 & 41 \\
\hline $65-69$ & 718 & 42 & 51 \\
\hline $70-74$ & 543 & 35 & 50 \\
\hline $75-79$ & 76 & 8 & 8 \\
\hline Total & $5745^{a}$ & 172 & 210 \\
\hline
\end{tabular}

Note. Cumulative LC standardized mortality ratio was 0.82 (95\% CI 0.67-0.99); $p=0.048$.

a Cohort individuals alive as of January 1, 1999. LC, lung cancer; CI, confidence interval. surprise, as the subjects targeted for screening in our study were quite different from those of the PLCO. While we investigated the totality of screening-eligible ever-smokers resident in 50 communities of the Varese Province, characterized by high LC incidence (5.15/1000 person-years) [11], the PLCO trial was not specifically designed to investigate smokers [25] and recruited highly selected volunteers, as reflected by the fact that enrolment averaged approximately $1 \%$ of the invited population [26]. Moreover, in the PLCO about $80 \%$ of the total of intervention group LCs were nonscreen-detected and could be diluting any screening effect [5]. While in theory a population-based randomized trial is the ideal method to assess the efficacy of an LC screening intervention [27], in practice the historical randomized CXR screening trials have subsequently been associated with important methodological weaknesses $[1,4,7,8]$. In addition, the recently reported findings of the PLCO randomized radiographic screening are difficult to generalize to smokers, as about half of participants were never smokers [5]. Observational studies of CXR screening in all eligible smokers of the population, with appropriate comparator groups, may contribute important information with regard to the effectiveness of screening in the community setting. In our observational study, $21 \%$ of the invited population-based cohort chose to participate in screening. This attendance rate is similar to the 20-33\% participation recorded in Japanese mass screenings with CXR [28,29], and indicates a generally limited response to LC screening invitation. Because the assessment of screening efficacy may be biased by selection of participants [11], in our study we evaluated the overall impact of screening on LC mortality in all the invitees. The assessment of SMR according to invitation-to-screen has been previously used for the evaluation of population-based cancer screening, in order to eliminate self-selection bias of participants [14,15]. Other investigators, using the SMR to compare the LC mortality experience in a CT screened cohort of smokers in New York State and in two large unscreened cohorts, reported significant reductions in deaths from LC, documented by SMR respectively of 0.64 and 0.36 [13]. In our study the cumulative LC SMR of the CXR screening invited cohort was 0.82 , a result that must be interpreted cautiously. Because the design of our study was observational, we assumed that the cohort and the reference population were identical in all important variables except the intervention, or that we could correct for the relevant differences. The LC SMR may reflect the effectiveness of our screening programme only if the LC mortality risk profile in the cohort and in the reference population were identical at baseline, and if the two groups received the same standard of care except for screening in the cohort. As to comparability, it is noteworthy that the cohort and the reference population were contemporary and were extracted by the same smoking history criteria (Table 2) from the Varese Province general population of both genders, of birth cohort 1923-1953, resident in the 44town catchment area [11]. Under these circumstances, it is highly unlikely that our observational study was vulnerable to confounding by important differences between the cohort and the reference population, except for confounding caused by the absence of subjects unfit for surgery or with LC symptoms in the cohort at baseline. To correct for this healthier-cohort bias that is in favour of screening effectiveness, we excluded from LC mortality analysis the initial 18 months of study, as suggested by other authors [18]. Because the time from LC symptoms to death is rarely longer than a few months, the healthier-cohort bias of the cumulative LC SMR assessed during the subsequent period 1999-2006 was likely negligible. As to the completeness of follow-up and of LC deaths data collection, it must be noted that the vital status ascertainment rates at study cut off were very high, and nearly identical in the cohort and in the source population (98.5\% and $98.2 \%$, respectively). Notably, contamination by CXR screening that was performed in approximately $9 \%$ of the reference population (1244 participants of the cohort and 1221 
"uninvited participants" referred to in the methods) possibly biased the LC SMR modestly against screening effectiveness. Strengths of this study are $>98 \%$ complete follow-up the cohort, and intentto-screen analysis of LC mortality. An additional strength is the conservative assessment of LC SMR. To this effect, in order to calculate the expected number of LC deaths in a worst case scenario, we used the lowest value (0.86) in the range of smoking-attributable proportion of LCS.

While fully acknowledging the limitation of this nonrandomized population-based cohort study with a comparison group, our findings suggest causality between implementation of the CXR screening programme and significant reduction of LC mortality in the invitation-to-screen cohort. It is noteworthy that the LC SMR of 0.82 estimated after CXR screening is consistent with the relative stage shift of LC and with the significantly enhanced long-term (10year) LC-specific survival in the invitation-to-screen cohort, that we recently reported [12]. It is speculative whether the GPs collaborating in this study had an increased LC awareness and provided better than usual care to the whole cohort they invited to the screening programme, possibly contributing to lower the LC mortality. We also hypothesize that an increased LC awareness among screening participants [11] contributed to decrease LC mortality by improving cancer treatment, as suggested in the setting of breast cancer screening [30]. It is noteworthy that our screening programme was managed for nearly a decade without severe complications. We previously reported few false-positive screening tests (3.4\%), and few futile invasive diagnostic procedures (1.6/1000 tests), none of which caused serious adverse events. There were no deaths related to treatment of screen-detected LCs [12].

A complete costs-benefit analysis of our CXR screening programme of smokers at community level remains to be performed, to examine the possibility of whether the $18 \%$ LC mortality reduction that we estimated may be relevant for public health policy.

\section{Conflict of interest statement}

The authors declare no conflicts of interest.

\section{Acknowledgements}

This study was supported in part by University of Insubria Research Funds FAR1997-FAR2007 and by Lions Clubs Varese Host and District $108 \mathrm{Ib}-1$ annual grants 1997-2008.

We gratefully acknowledge the assistance provided by Amare no-profit organization-Como, Italy and Foundation Paola Giancola for Cancer Research-Como, Italy.

\section{References}

[1] Church TR. Chest radiography as the comparison for spiral CT in the National Lung Screening Trial. Acad Radiol 2003;10:713-5.

[2] Fontana RS, Sanderson DR, Woolner LB, Taylor WF, Miller WE, Muhm JR, et al. Screening for lung cancer: a critique of the Mayo lung project. Cancer 1991;67:1155-64.

[3] Kubik AK, Parkin DM, Zatloukal P. Czech study on lung cancer screening. Posttrial follow-up of lung cancer deaths up to year 15 since enrollment. Cancer 2000;89(Suppl):2363-8
[4] Manser R, Irving LB, Stone C, Byrnes G, Abramson M, Campbell D. Screening for lung cancer. Cochrane Database Syst Rev 2004, http://dx.doi.org/10.1002/14651858.

[5] Oken MM, Hocking WG, Kvale PA, Andriole GL, Buys SS, Church TR, et al. Screening by chest radiograph and lung cancer mortality. The prostate, lung, colorectal, and ovarian (PLCO) randomised trial. JAMA 2011;306:1865-73.

[6] Bach PB, Silvestri GA, Hanger M, Jett J. Screening for lung cancer: ACCP evidencebased clinical practices guidelines (2nd ed.). Chest 2007;132(Suppl):69-77.

[7] Dominioni L, Poli A, Mantovani W, Rotolo N, Imperatori A. Volunteer effect and compromised randomization in the Mayo Project of screening for lung cancer. Eur J Epidemiol 2011;26:79-80.

[8] Sox HC. Screening for lung cancer with chest radiographs. JAMA 2011;306:1916-8.

[9] U.S. Preventive Services Task Force. Lung cancer screening: recommendation statement. Ann Intern Med 2004;140:738-9.

[10] Sagawa M, Nakayama T, Tsukada H, Nishii K, Baba T, Kurita Y, et al. The efficacy of lung cancer screening conducted in 1990s: four case-control studies in Japan. Lung Cancer 2003;41:29-36.

[11] Dominioni L, Rotolo N, Poli A, Paolucci M, Sessa F, D’Ambrosio V, et al. Self-selection effects in smokers attending lung cancer screening. A 9.5-year population-based cohort study in Varese, Italy. J Thorac Oncol 2010;5:428-35.

[12] Dominioni L, Rotolo N, Mantovani W, Poli A, Pisani S, Conti V, et al. A populationbased cohort study of chest X-ray screening in smokers: lung cancer detection findings and follow-up. BMC Cancer 2012;12:18.

[13] Henschke CI, Boffetta P, Gorlova O, Yip R, Delancey JO, Foy M. Assessment of lung-cancer mortality reduction from CT screening. Lung Cancer 2011;71:328-32.

[14] Hakama M, Pukkala E, Soderman B, Day N. Implementation of screening as a public health policy: issue in design and evaluation. J Med Screen 1999;6:209-16.

[15] Puliti D, Zappa M. Breast cancer screening: are we seeing the benefit? BMC Med 2012;10:106.

[16] Friedman GD, Collen MF, Fireman BH. Multiphasic health checkup evaluation: a 16-year follow-up. J Chron Dis 1986;39:453-63.

[17] Imperatori A, Harrison RN, Leitch DN, Rovera F, Lepore G, Dionigi G, et al. Lung cancer in Teesside (UK) and Varese (Italy): a comparison of management and survival. Thorax 2006;61:232-9.

[18] Bach PB, Jett JR, Pastorino U, Tockman MS, Swensen SJ, Begg CB. Computed tomography screening and lung cancer outcomes. JAMA 2007;297:953-61 [Erratum in: JAMA 2007;298:518].

[19] Kim HJ, Fay MP, Feuer EJ, Midthune DN. Permutation tests for joinpoint regression with applications to cancer rates. Stat Med 2000;19:335-51 [Erratum in: Stat Med 2001;20:655].

[20] Imperatori A, Rotolo N, Conti V, Di Natale D, Tropeano V, Mantovani W. Prevalence of heavy smokers in the year 2000 in the Province of Varese, Italy. Monaldi Arch Chest Dis 2010;73:152-4.

[21] Regione Lombardia. Servizi Informativi Socio-Sanitari. Available at: http://was.cgi.crs.lombardia.it/SebsGate/ [accessed September 24, 2012].

[22] Associazione Italiana Registri Tumori. Varese Cancer Registry. Available at: http://www.registri-tumori.it/ [accessed September 24, 2012].

[23] Azienda Sanitaria Locale della Provincia di Varese. Varese Province epidemiology observatory. Available at: http://www.asl.varese.it/ [accessed September 24, 2012].

[24] National Cancer Institute. Joinpoint regression programme, version 3.4.2. Available at: http://www.srab.cancer.gov/joinpoint/ [accessed September 24, 2012]

[25] Prorok PC, Andriole GL, Bresalier RS, Buys SS, Chia D, Crawford ED, et al. Design of the prostate, lung, colorectal and ovarian (PLCO) cancer screening trial. Control Clin Trials 2000;21(Suppl):273-309.

[26] Gren L, Broski K, Childs J, Cordes J, Engelhard D, Gahagan B, et al. Recruitment methods employed in the prostate, lung, colorectal, and ovarian cancer screening trial. Clin Trials 2009;6:52-9.

[27] Prorok PC, Chamberlain J, Day NE, Hakama M, Miller AB. UICC workshop on the evaluation of screening programmes for cancer. Int J Cancer 1984;34:1-4.

[28] Nishii K, Ueoka H, Kiura K, Kodani T, Tabata M, Shibayama T, et al. A casecontrol study of lung cancer screening in Okayama Prefecture, Japan. Lung Cancer 2001;34:325-32.

[29] Okamoto N, Suzuki T, Hasegawa H, Gotoh T, Hagiwara S, Sekimoto M, et al. Evaluation of a clinic-based screening program for lung cancer with case-control design in Kanagawa, Japan. Lung Cancer 1999;25:77-85.

[30] Jorgensen KJ, Keen JD, Gotzsche PC. Is mammographic screening justifiable considering its substantial overdiagnosis rate and minor effect on mortality? Radiology 2011;260:621-7. 